\title{
Mechanism of Influenza A M2 Ion-Channel Inhibition: A Docking and QSAR Study
}

\author{
Alexander V. Gaiday ${ }^{1}$, Igor A. Levandovskiy ${ }^{1}$, \\ Kendall G. Byler ${ }^{2}$, and Tatyana E. Shubina ${ }^{1,2}$ \\ ${ }^{1}$ Department of Organic Chemistry, Kiev Polytechnic Institute, \\ pr. Pobedy 37,03056 Kiev, Ukraine \\ ${ }^{2}$ Computer-Chemie-Centrum der Friedrich-Alexander Universität \\ Erlangen-Nürnberg, Nägelsbachstraße 25, \\ 91052 Erlangen, Germany \\ tatyana.shubina@chemie.uni-erlangen.de
}

\begin{abstract}
Binding of blockers to the Influenza A ion-channel is studied using automated docking calculations. Our study suggests that studied cage compounds inhibit the M2 ion channel by binding to the His37 residue. The adamantane cage fits into a pocket formed by Trp41 residue, while the hydrogen bond is formed between hydrogen atom of ammonium nitrogen and the nitrogen of histidine residue. This finding is supported by experimental data and should help to obtain better understanding of the inhibition mechanism of the Influenza A M2 ion channel.
\end{abstract}

Keywords: Influenza A, ion-channel inhibition, QSAR, antiviral drugs, cage compounds.

\section{Introduction}

The influenza A virus, with 27 known subtypes that range from low to high pathogenicity in the bird population, also infects humans and other mammals. Subtype H5N1, currently known as avian or bird flu, is of particular interest because of its increasing pathogenicity and its ability to form a new viral subtype to which there is no native immunity in human hosts.

The influenza virus is bound by a membrane taken from the plasma membrane of an infected cell. The M2 protein is present in this membrane and forms protonselective channels that are essential to viral function. Based on experimental data, it is assumed that the M2 proton channel consists of a tetrameric array of parallel TM peptides with their $N$-termini directed towards the outside of the virus and has following amino acids sequence: $N$-term- ${ }^{26}{ }^{26}$ VAAS $^{31}$ IIGI LHLILWIL ${ }^{43}-C$-term. [1, 2, 2, 3, 4, It is also believed that residues Val27, Ala30, Ser31, Gly34, His37, Lys38, and Trp41 form the pore lining; and it is also possible that histidine- 37 plays an important role in the gating of the channel, or in the mechanism of $\mathrm{H}^{+}$transport or both. 
Adamantane derivatives have been used successfully for the prevention and treatment of influenza A virus infection for more than 30 years. [5], [6], [7] It is proposed that these drugs inhibit influenza A virus replication by blocking the M2-protein ion channel. This prevents fusion of the viruses with the host-cell membranes and the release of viral RNA into the cytoplasm of infected cells. 8 There is experimental evidence that the amantadine blockade of the M2 channel occurs within the TM region. However, the mechanism of this process is still unclear.

It was recently discovered that the Influenza A virus now exhibits resistance to both, amantadine and rimantadine. 9] This resistance to adamantane derivatives is believed to be due to a serine-31-to-asparagine mutation in the M2-protein. 10. Also, it is possible that an amino acid substitution at positions 26, 27, 30 or 34 in the transmembrane region of the M2 protein is also responsible for resistance to these drugs. [7, [11]

In such cases, treatment by amantadine and rimantadine will be less predictably effective for treatment or prophylaxis in the event of a pandemic outbreak of influenza.

Obviously, better understanding the exact nature of M2 proton channel inhibition by amantadine and other possible drug candidates is needed. The present study aims at shedding some light into this problem.

\section{Results and Discussion}

Cyclopentylamine (1), cyclohexylamine (2), amantadine (3), rimantadine (6) and other adamantane derivatives (Fig. 1) in their protonated states were initially optimized at the B3LYP/6-31G(d) level [12, 13, of theory within the Gaussian03 program package. 14]

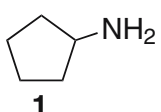

1<smiles>NC1CCCCC1</smiles>

2<smiles>[R]C12CC3CC([R7])(C1)CC(N)(C3)C2</smiles>

3: $\mathrm{R}_{1}=\mathrm{R}_{2}=\mathrm{H}$

4: $\mathrm{R}_{1}=\mathrm{H}, \mathrm{R}_{2}=\mathrm{CH}_{3}$

5: $\mathrm{R}_{1}=\mathrm{R}_{2}=\mathrm{CH}_{3}$<smiles>[R]C12CC3CC([R2])(C1)CC(C(C)N)(C3)C2</smiles>

6: $\mathrm{R}_{1}=\mathrm{R}_{2}=\mathrm{H}$ 7: $\mathrm{R}_{1}=\mathrm{H}, \mathrm{R}_{2}=\mathrm{CH}_{3}$

8: $R_{1}=R_{2}=\mathrm{CH}_{3}$<smiles>[R2]C12CC3CC([R])(C1)CC(NC(C)=O)(C3)C2</smiles>

9: $\mathrm{R}_{1}=\mathrm{R}_{2}=\mathrm{H}$

10: $\mathrm{R}_{1}=\mathrm{H}, \mathrm{R}_{2}=\mathrm{CH}_{3}$

11: $\mathrm{R}_{1}=\mathrm{R}_{2}=\mathrm{CH}_{3}$<smiles>[R2]C12CC3CC([R7])(C1)CC(C(C)NC(C)=O)(C3)C2</smiles>

12: $\mathrm{R}_{1}=\mathrm{R}_{2}=\mathrm{H}$

13: $\mathrm{R}_{1}=\mathrm{H}, \mathrm{R}_{2}=\mathrm{CH}_{3}$

14: $\mathrm{R}_{1}=\mathrm{R}_{2}=\mathrm{CH}_{3}$<smiles>[R]N([R])C12CC3CC(CC(C3)C1)C2</smiles>

15: $\mathrm{R}_{1}=\mathrm{H}, \mathrm{R}_{2}=\mathrm{CH}_{3}$

16: $\mathrm{R}_{1}=\mathrm{R}_{2}=\mathrm{CH}_{3}$

Fig. 1. Compounds 1-16 used in this work 
Table 1. Computed docking energies and estimated binding constants of the compounds 1-16

\begin{tabular}{|c|c|c|c|c|}
\hline \multirow[b]{2}{*}{$\mathrm{N}$} & \multicolumn{3}{|c|}{$\begin{array}{c}\text { Docking energy, -E, kcal/mol } \\
\text { Binding pocket }\end{array}$} & $\begin{array}{c}\text { Inhibition constant }\left(K_{i}\right) \\
\text { Binding pocket }\end{array}$ \\
\hline & $\mathrm{I}$ & II & III & III \\
\hline 1 & 3.79 & 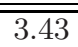 & 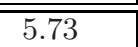 & $\begin{array}{ll}0.00 & 2.48 \cdot 10^{-5}\end{array}$ \\
\hline 2 & 3.87 & 4.34 & 6.56 & $6.17 \cdot 10^{-4} 2.81 \cdot 10^{-4} 6.44 \cdot 10^{-6}$ \\
\hline 3 & 5.09 & 6.00 & 8.73 & $5.68 \cdot 10^{-5} 1.13 \cdot 10^{-5} 1.29 \cdot 10^{-7}$ \\
\hline 4 & 5.27 & 6.31 & 8.39 & $4.78 \cdot 10^{-5} 8.25 \cdot 10^{-6} 2.45 \cdot 10^{-7}$ \\
\hline 5 & 5.65 & 6.87 & 9.15 & $1.05 \cdot 10^{-5} 1.87 \cdot 10^{-6} 3.98 \cdot 10^{-8}$ \\
\hline 6 & 5.84 & 6.74 & 9.84 & $1.66 \cdot 10^{-5} 3.86 \cdot 10^{-6} 1.97 \cdot 10^{-8}$ \\
\hline 7 & 6.34 & 7.20 & 10.23 & $7.34 \cdot 10^{-6} 1.40 \cdot 10^{-6} 8.16 \cdot 10^{-9}$ \\
\hline 8 & 6.87 & 7.89 & 10.63 & $2.32 \cdot 10^{-6} 4.53 \cdot 10^{-7} 3.52 \cdot 10^{-9}$ \\
\hline 9 & 5.67 & 6.27 & 9.51 & $1.34 \cdot 10^{-5} 5.02 \cdot 10^{-6} 2.21 \cdot 10^{-8}$ \\
\hline 10 & 6.96 & 8.09 & 10.31 & $4.03 \cdot 10^{-6} 5.75 \cdot 10^{-7} 1.38 \cdot 10^{-8}$ \\
\hline 11 & 6.58 & 7.28 & 10.02 & $3.27 \cdot 10^{-6} 9.93 \cdot 10^{-7} 8.06 \cdot 10^{-9}$ \\
\hline 12 & 6.67 & 1.03 & 10.95 & $7.71 \cdot 10^{-6} 1.84 \cdot 10^{-6} 5.74 \cdot 10^{-9}$ \\
\hline 13 & 6.96 & 8.09 & 10.31 & $4.03 \cdot 10^{-6} 5.75 \cdot 10^{-7} 1.33 \cdot 10^{-8}$ \\
\hline 14 & 7.30 & 8.60 & 10.99 & $2.17 \cdot 10^{-6} 3.24 \cdot 10^{-7} 5.25 \cdot 10^{-9}$ \\
\hline 15 & 5.02 & 5.64 & 8.61 & $2.29 \cdot 10^{-5} 8.64 \cdot 10^{-6} 6.01 \cdot 10^{-8}$ \\
\hline 16 & 4.38 & 5.26 & 8.11 & $1.61 \cdot 10^{-5} 3.40 \cdot 10^{-6} 2.66 \cdot 10^{-8}$ \\
\hline
\end{tabular}

For modelling the transmembrane part of the M2 channel we used the PDB entry 1 NYJ with the following amino acid sequence order: SSDPLVVAASIIGILHLILWILDRL.

The blockers and the protein were prepared for docking using ADT, the AutoDock tool graphical interface. [15] For each ligand Gasteiger charges were assigned and atomic solvatation parameters were added. For the protein, polar hydrogen charges of Kollman-type were assigned and the non-polar hydrogens were merged with the carbons. The internal degrees of freedom and torsions were set for each ligand.

The AutoDock3.0 software [16] was used to obtain unbiased binding positions, estimate docking energies and inhibition constants for the studied compounds 1-16. The search was carried out with the Lamarckian Genetic Algorithm: a starting population of 150 ligand conformations with a stopping criteria of a maximum 10 millions energy evaluations. Evaluation of the results was done by sorting the different complexes with respect to the predicted binding energy. A cluster analysis based on RMSD values, with reference to the starting geometry, 


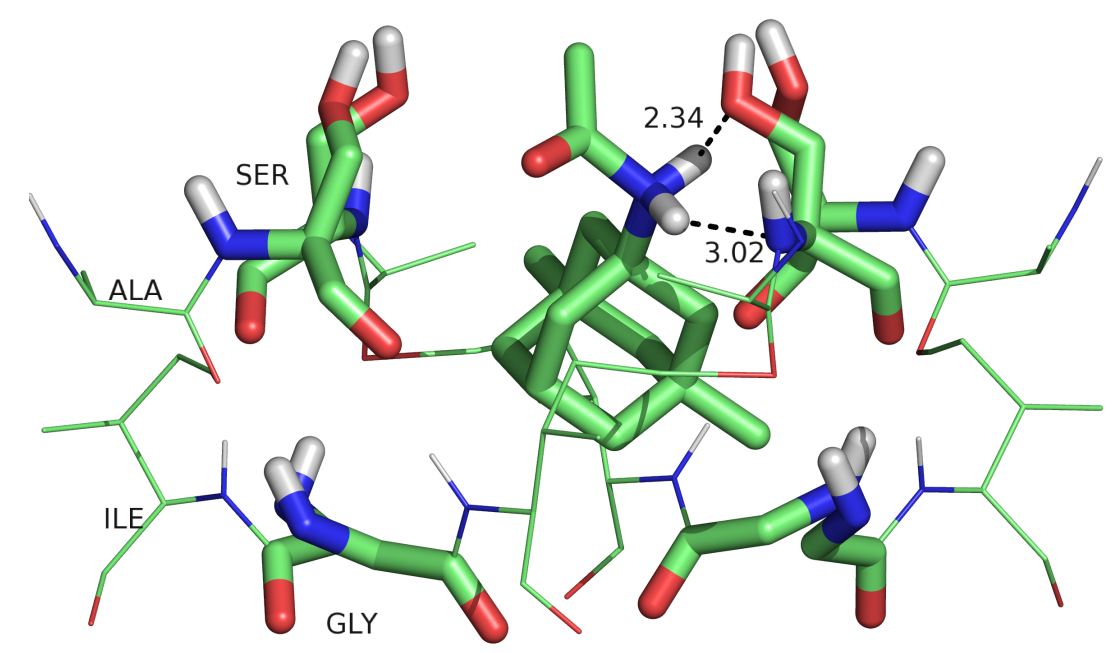

Fig. 2. A detailed view of ligand 14 docked into binding pocket $\mathbf{I}$. For clear view only Ala30-Ser31-Gly34-Ile35 residues are shown in sticks. Bond lengths in A.

was subsequently performed. All the figures in this paper were produced with PyMOL. 17]

The results of the docking study shows that the TM part of the M2 channel can be treated as three different binding pockets within large pore. The first binding pocket $(\mathbf{I})$ is defined by Val27-Ala30-Ser31 residues of each $\alpha$-helixes. It is believed that amantadine resistance is determined by amino acid exchanges within this region. Also, in an early molecular modelling study [18] Sansom et al. suggested localization of the amantadine binding site close to Ser31. In the formation of the second binding pocket (II) participate mostly Gly34 and Ile35. Finally, His37 residue is responsible for the formation of the third binding pocket (III).

The computed docking energies of $\mathbf{1 - 1 6}$ within each binding pocket and computed respective inhibition constants $\left(K_{i}\right)$ are summarized in Table 1 . Both cyclic amines $\mathbf{1}$ and $\mathbf{2}$ exhibit the lowest binding energies to all three binding sites. Our study confirms that cyclopentylamine $\mathbf{1}$ does not block the M2 channel due to low binding energy and, thus, probable ability to pass through the pore, which is in agreement with its lack of antiviral activity in infected cells. [19], [20] While cyclohexylamine $\mathbf{2}$ is more bulky than cyclopentylamine $\mathbf{1}$, it still shows low inhibition properties. This correlates with the sensitivity of influenza virus replication to the size of aliphatic ring in the cyclic amines.

For all studied compounds His37 binding site (III) was found to be the most preferred one. Rimantadine 6 has higher binding energies and exhibits ca. 10-fold higher inhibition properties than amantadine 3. This can be explained by the presence of the $\mathrm{CH}$ group between amino group and adamantane cage, which allows rotation of the amino group and, therefore, assists closer and more favorable interaction with amino acid residues of the proton channel compared to amantadine. 


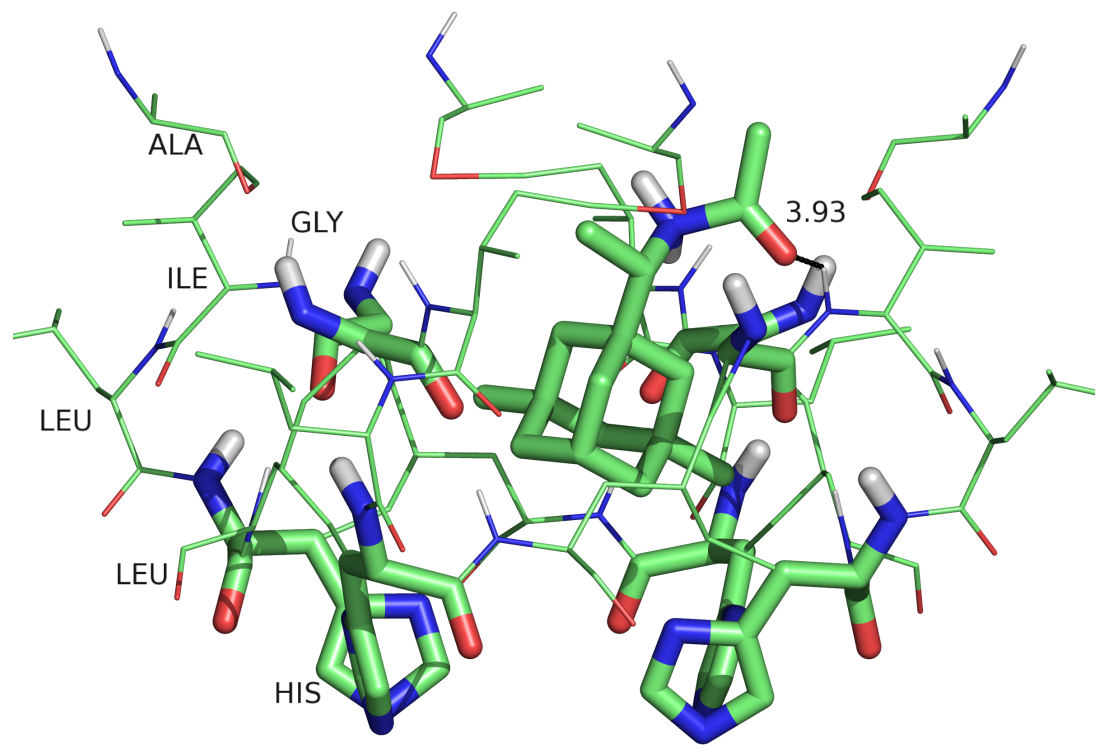

Fig. 3. A detailed view of ligand $\mathbf{1 4}$ docked into binding pocket II. For clear view only Ala30-Gly34-Ile35-His37 residues are shown in sticks. Bond lengths in $\AA$.

Consequent substitution of hydrogens in 3 - and 5 - positions in the adamantane cage into methyl groups leading to compounds $4,7,10,13$ and $5,8,11,14$ increases binding energies and inhibition properties of $\mathbf{4 ,} \mathbf{5}, \mathbf{7}-\mathbf{1 4}$ (Table 1).

Additional consequent introduction of methyl groups in adamantane cage of compounds 9 and 12 leading to compounds 10,13 and 11, 14 also increases binding energies and inhibition properties as it was in case of amantadine and rimantadine (Table 1). In fact, increase of molecular volume of the inhibitor and presence of the acetyl group makes $\mathbf{1 4}$ the strongest blocker among studied compounds.

It should be mentioned that the nitrogen atom of amino group of all four ligands forms a hydrogen bond with the residues that form the binding pocket. Figures 24 show a detailed view of the docked representative ligands 14 or 8 within the first, second and third binding pockets, respectively. The distance between the ammonium hydrogen and the oxygen atom of serine residue in the first binding pocket ranges from $2.37 \AA$ (in $\mathbf{2}$ ), $2.0 \AA$ (in $\mathbf{1}$ and $\mathbf{3}$ ) to $1.84 \AA$ (4).

In the second binding pocket there is also possibility to form hydrogen bonds between the hydrogen atom of $\mathrm{NH}_{3}$ group and the oxygen atom of glycine.

Nevertheless, for all ligands studied here the most preferred binding pocket is the third one. Interestingly enough, opposite to our findings, Sansom et al.18 showed that near the His37 residue there is a substantial barrier in case of amantadine and favorable interaction with cyclopentylamine. Our docking study shows that the aliphatic rings and adamantane cage fit into a pocket formed by tryptophan-34 (Fig. 4), the hydrogen bond is formed between hydrogen of $\mathrm{NH}_{3}$ 
group and the nitrogen of histidine residue, leading to more efficient blocking of the channel. Moreover, the difference in the estimated inhibition constants of amantadine $(3)$ and rimantadine (4) docked into the binding pocket III agrees fortuitously with the experimentally observed order. These findings are in agreement with recently proposed model [21] in which the ammonium nitrogen of amantadine shares a hydrogen bond with an unprotonated histidine-37 imidazole while the adamantyl group fits in the lipophilic pocked formed in the vicinity of Gly34.

It is also worth to note that a similar adamantane- $\mathrm{NH}_{3}-\mathrm{His}$ interaction has been also recently found [22] for the Hepatitis $\mathrm{C}$ virus ion channel p7.

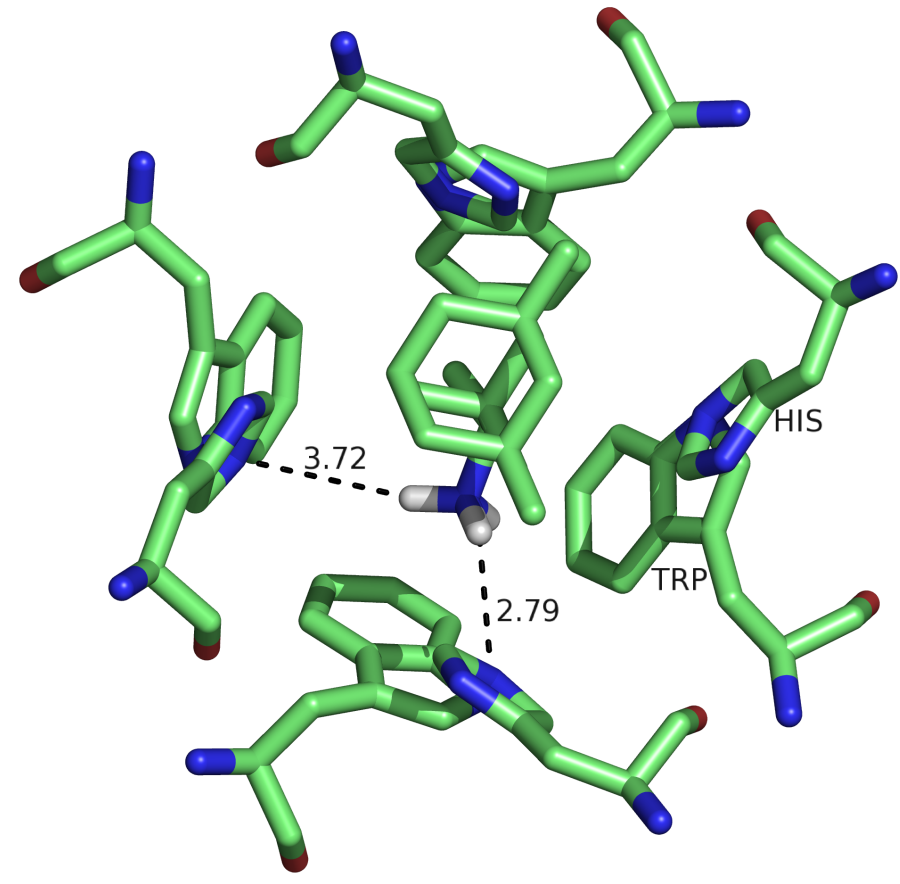

Fig. 4. A detailed view of ligand $\mathbf{8}$ docked into binding pocket III. Only His37 and Trp41 are shown in sticks Bond lengths in $\AA$.

The structures in the influenza data set were aligned using SYBYL 7.0 program. Semi-empirical single-point calculations with the AM1 Hamiltonian were performed on each using VAMP 9.023 to calculate charges and orbital information. A three-dimensional grid with a point spacing of $2 \AA$ and a $4 \AA$ border was generated used in the calculation of seven local properties: electron density $\mathrm{d}_{e}$, electron affinity $\mathrm{EA}_{L}$, electronegativity $\mathrm{c}_{L}$, hardness $\mathrm{h}_{L}$, ionization potential $\mathrm{IE}_{L}$, electrostatic potential $\mathrm{V}$, and polarizability $\mathrm{a}_{L}$ at each grid point using Parasurf 07 program package 24.

Points interior to the molecular surface were removed from the grid by using a generalized van der Waals radius of $1.16 \AA$ in order to ensure that property values 
that bear no direct relation to surface activity would not appear in the PLS analyses. This atomic radius, which is slightly smaller than the van der Waals radius for the hydrogen atom $(1.20 \AA)$, was chosen in order to leave enough surface electron density to use the local electron density as a steric parameter in the 3D-QSAR analysis. The local property values at the grid points were then used as independent variables in separate partial least squares regressions, using associated physical property values as target values.

The partial least squares analyses were performed using an in-house program using the SIMPLS algorithm and a full cross-validation scheme, re-centering and re-scaling the included data for each run. The PLS regressions were carried out initially to ten vector components in order to determine the maximum number of components to be included in the final model, using the cross-validated standard error of prediction (SEP) of the model to choose the appropriate number of components. In the cases where PLS analyses were performed using single local properties, the property data was normalized by the mean. The regression coefficients for the final model were then used to generate a three-dimensional representation of the property space with colored spheres using Pymol. 17] Those grid points with a positive relationship to the particular target property are color-coded red, while those with a negative relationship to the target property are color-coded blue. In addition to color-coding, the size of the grid spheres, determined by the standardized magnitude of the regression coefficients, represents the magnitude of the relationship between the local property at that point to the target value.

The PLS analysis based on SYBYL CoMFA yelds with an $\mathrm{r}^{2}=0.993$ for 10 components, however, reducing number of components to 4 leads to:

$$
\mathrm{r}^{2}=0.909 ; \mathrm{q}^{2}=0.348 ; \sigma=3.46
$$

$78.2 \%$ was found to be responsible for the steric influence and $21.8 \%$ - for the electrostatic interaction.

QSAR performed with Parasurf07 (with 3 components) gives somewhat better correlation:

$$
\mathrm{r}^{2}=0.913 ; \mathrm{q}^{2}=0.401 ; \sigma=3.73
$$

The 3D-QSAR results for the two most contributed properties - electron density $\mathrm{d}_{e}$ and ionization potential $\mathrm{IE}_{L}$ are shown below (Fig. 5 left and right, respectively).

In this work, we have reported modeling of the M2-TM channel inhibition by different ligands. We have demonstrated the difference in binding mechanism between small cyclic amines (1-2) and adamantane derivatives (3-16) in the M2 proton channel.

The docking study shows that the M2 proton channel is blocked by cage compounds and suggests that the preferred binding site is in a vicinity of His37 

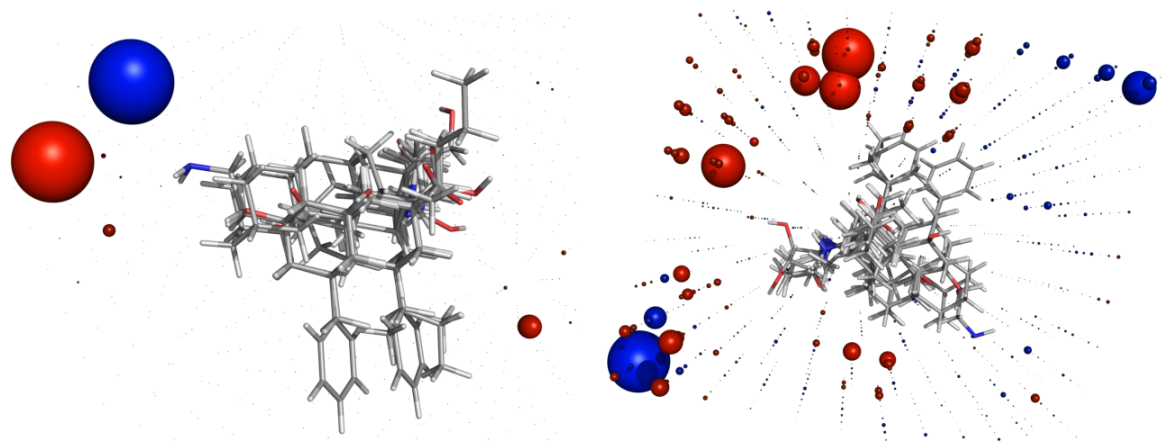

Fig. 5. Electron density $\mathrm{d}_{e}$ (left) and ionization potential $\mathrm{IE}_{L}$ (right)

residue. The calculated inhibiting ability of amantadine and rimantadine reproduces the relations observed clinically.

Based on our docking study with 3D-QSAR results we suggest that next generation of potential M2 inhibitors should include lipophilic adamantane or other hydrocarbon cage (i.e. trishomocubane) combined with polar groups (e.g. hydroxyl, carboxyl, urea/thiourea moiety) separated from cage by methylene bridges. Nevertheless, more clearer understanding of the nature of inhibition mechanism is needed. One way of doing it - via molecular dynamic simulation of the whole M2 ion-channel and its interaction with inhibitors. Results of such study will be published elsewhere.

Acknowledgments. This work was supported by grants for the young scientists from the President of Ukraine (GP 2006, 2007 and 2008).

\section{References}

1. Lamb, R.A., Holsinger, L.J., Pinto, L.H., Wimmer, E. (eds.): vol. 28, p. 526. Cold Spring Harbor Lab Press, New York (1994)

2. Hay, A.J.: Semin. Virol 3, 21 (1992)

3. Chizhmakov, I.V., Geraghty, F.M., Ogden, D.C., Hayhurst, A., Antoniou, M., Hay, A.J.: J. J. Physiol. 494, 329 (1996)

4. Shimbo, K., Brassard, D.L., Lamb, R.A., Pinto, L.H.: Biophys. 70, 1335 (1996)

5. Tominack, R.L., Hyden, F.G.: Rimantadine hydrochloride and amantadine hydrochloride use in Influenza A virus infection. Infect. Dis. Clin. North. Am. 1, 459 (1987)

6. Dolin, R., Reichman, R.C., Madore, H.P., Maynard, R., Linton, P.N., WebberJones, J.: A controlled trial of amantadine and rimantadine in the prophylaxis of Influenza A virus. N. Engl. J. Med. 307, 580 (1982)

7. Belshe, R.B., Smith, M.H., Hall, G.B., Bettis, R., Hay, A.J.: Genetic basis of resistance to rimantadine emerging during treatment of influenza virus infection. J. Virol. 62, 1508 (1988)

8. Wang, C., Takeuchi, K., Pinto, L.H., Lamb, R.A.: Ion channel activity of Influenza A virus M2 protein: characterization of the amantadine block. J. Virol. 67, 5585 (1993) 
9. Evolution of H5N1 avian influenza viruses in Asia, http://www.cdc.gov/ncidod/EID/vol11no10/pdfs/05-0644.pdf

10. Puthavathana, P., Auewarakul, P., Charoenying, P.C.: Molecular characterization of the complete genome of human influenza H5N1 viruses isolated from Tailand. J. Gen. Virol. 86, 423 (2005)

11. Hay, A.J., Zambon, M.C., Wolstenholme, A.J., Skehel, J.J., Smith, M.H.: Molecular basis of resistance of Influenza A viruses to amantaine. J. Antimicrob. Chemother. 8(suppl. B), 19 (1986)

12. Becke, A.D.: J. Chem. Phys. 98, 5648 (1993)

13. Lee, C., Yang, W., Parr, R.G.: Phys. Rev. 37, 785 (1988)

14. Frisch, M.J., Trucks, G.W., Schlegel, et al.: Gaussian 03, Revision B.03, Gaussian, Inc., Wallingford CT (2004)

15. Sanner, M., Duncan, B., Carillo, C., Olson, A.: Integrating computation and visualization for biomolecular analysis: an example using python and avs. Pac Symp Biocomput, 401-412 (1999)

16. Morris, G.M., Goodsell, D.S., Halliday, R.S., Huey, R., Hart, W.E., Belew, R.K., Olson, A.: Automated docking using a lamarckian genetic algorithm and empirical binding free energy function. J. Comp. Chem. 19, 1639 (1998)

17. DeLano, W.L.: The pymol molecular graphics system (2002), http://www . pymol.org

18. Sansom, M.S., Kerr, I.D.: Influenza virus M2 protein: a molecular modelling study of the ion channel. Protein Engineering 6, 65 (1993)

19. Hay, A.J., Wolstenholme, A.J., Skehel, J.J., Smith, M.H.: EMBO Journal 4, 3021 (1985)

20. Hay, A.J., Zambon, M.: Multiple actions of amantadine against influenza virus, volume Antiviral Drugs and Interferon: The Molecular basis of their activity (1984)

21. Mould, J.A., Li, H.-C., Dudlak, C.S., Lear, J.D., Pekosz, A., Lamb, R.A., Pinto, L.H.J.: J. Biol. Chem. 275, 8592 (2000)

22. Ptagrias, G., Zitzmann, N.D., Fischer, W.B.: J. Med. Chem. 49, 648 (2006)

23. Clark, T., Alex, A., Beck, B., Chandrasekhar, J., Gedeck, P., Horn, A.H.C., Hutter, M., Martin, B., Rauhut, G., Sauer, W., Schindler, T., Steinke, T.: VAMP 9.0. Computer-Chemie-Centrum, Universität Erlangen-Nürnberg, Erlangen (2005)

24. ParaSurf 2007 CEPOS InSilico Ltd (2007) 